\title{
Odina Sturzenegger-Benoist
}

Maître de conférences en anthropologie, Université Paul Cézanne d’Aix-Marseille III

\section{(2008)}

\section{"Le métissage invisible.}

Un document produit en version numérique par Jean-Marie Tremblay, bénévole, professeur de sociologie au Cégep de Chicoutimi

Courriel: jean-marie tremblay@uqac.ca

Site web pédagogique : http://www.uqac.ca/jmt-sociologue/

Dans le cadre de: "Les classiques des sciences sociales" Une bibliothèque numérique fondée et dirigée par Jean-Marie Tremblay, professeur de sociologie au Cégep de Chicoutimi Site web: http://classiques.uqac.ca/

Une collection développée en collaboration avec la Bibliothèque Paul-Émile-Boulet de l'Université du Québec à Chicoutimi

Site web: http://bibliotheque.uqac.ca/ 


\section{Politique d'utilisation de la bibliothèque des Classiques}

Toute reproduction et rediffusion de nos fichiers est interdite, même avec la mention de leur provenance, sans l'autorisation formelle, écrite, du fondateur des Classiques des sciences sociales, Jean-Marie Tremblay, sociologue.

Les fichiers des Classiques des sciences sociales ne peuvent sans autorisation formelle:

- être hébergés (en fichier ou page web, en totalité ou en partie) sur un serveur autre que celui des Classiques.

- servir de base de travail à un autre fichier modifié ensuite par tout autre moyen (couleur, police, mise en page, extraits, support, etc...),

Les fichiers (.html, .doc, .pdf., .rtf, .jpg, .gif) disponibles sur le site Les Classiques des sciences sociales sont la propriété des Classiques des sciences sociales, un organisme à but non lucratif composé exclusivement de bénévoles.

Ils sont disponibles pour une utilisation intellectuelle et personnelle et, en aucun cas, commerciale. Toute utilisation à des fins commerciales des fichiers sur ce site est strictement interdite et toute rediffusion est également strictement interdite.

L'accès à notre travail est libre et gratuit à tous les utilisateurs. C'est notre mission.

Jean-Marie Tremblay, sociologue

Fondateur et Président-directeur général, LES CLASSIQUES DES SCIENCES SOCIALES. 
Cette édition électronique a été réalisée par Jean-Marie Tremblay, bénévole, professeur de sociologie au Cégep de Chicoutimi à partir de :

Odina Sturzenegger-Benoist

[Maître de conférences en anthropologie, Université Paul Cézanne d’Aix-Marseille III]

“Le métissage invisible”.

Un texte publié dans l'ouvrage sous la direction de Virginie Baby-Collin et Delphine Mercier, Sud à Sud, Dynamiques sociales et spatiales Amérique latine/Méditerranée, pp 61-73. Aix-en-Provence, Publications de l’Université de Provence, 2008, 311 pp. Collection : Monde contemporain.

[Autorisation formelle accordée par l'auteure le 2 mai 2009, et confirmée par M. Jean Benoist, de diffuser cet article dans Les Classiques des sciences sociales.]

Courriel : odina.benoist@wanadoo.fr et oj.benoist@wanadoo.fr

Polices de caractères utilisée :

Pour le texte: Times New Roman, 12 points.

Pour les citations : Times New Roman, 12 points.

Pour les notes de bas de page : Times New Roman, 12 points.

Édition électronique réalisée avec le traitement de textes Microsoft Word 2004 pour Macintosh.

Mise en page sur papier format : LETTRE (US letter), 8.5’’ x 11’')

Édition numérique réalisée le 9 mai 2009 à Chicoutimi, Ville de Saguenay, province de Québec, Canada. 


\section{Odina Sturzenegger-Benoist}

Maître de conférences en anthropologie, Université Paul Cézanne d’Aix-Marseille III

\section{“Le métissage invisible.”}

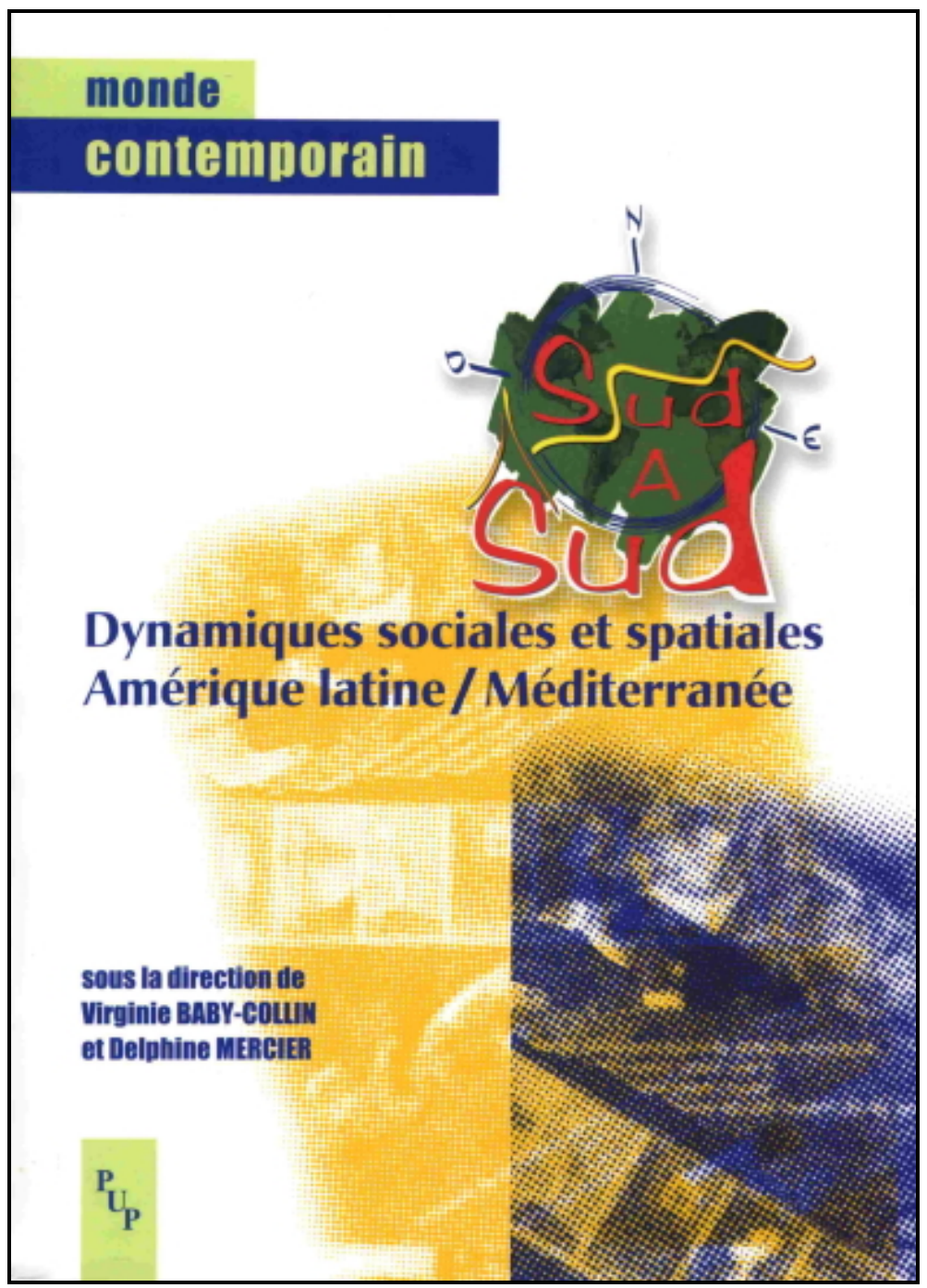

Un texte publié dans l'ouvrage sous la direction de Virginie Baby-Collin et Delphine Mercier, Sud à Sud, Dynamiques sociales et spatiales Amérique latine/Méditerranée, pp 61-73. Aix-en-Provence, Publications de l’Université de Provence, 2008, 311 pp. Collection : Monde contemporain. 


\section{Table des matières}

$\underline{\text { Introduction }}$

Le métissage : le contraste entre la définition biologique et la définition sociale Le métissage en Amérique espagnole

La société argentine: le souci de l'égalité et la question des apparences

Connotations du métissage

Connotations de la créolité

Quelques hypothèses

$\underline{\text { Références }}$ 
Odina Sturzenegger-Benoist

Maître de conférences en anthropologie, Université Paul Cézanne d’Aix-Marseille III

“Le métissage invisible”.

Un texte publié dans l'ouvrage sous la direction de Virginie Baby-Collin et Delphine Mercier, Sud à Sud, Dynamiques sociales et spatiales Amérique latine/Méditerranée, pp 61-73. Aix-en-Provence, Publications de l’Université de Provence, 2008, 311 pp. Collection : Monde contemporain.

\section{INTRODUCTION}

$\underline{\text { Retour à la table des matières }}$

Nous allons nous intéresser ici à une société, celle de l’Argentine, où le métissage, bien que clairement perceptible aux yeux d'un Européen, a été rendu invisible aux nationaux sous l'effet d'un projet politique développé dans la seconde moitié du $\mathrm{XIX}^{\mathrm{e}}$ siècle. Ce projet était porteur d'une idéologie qui a éduqué le regard à ne pas rattacher à un métissage les différences dans la morphologie des individus, et en particulier dans le teint de leur peau ${ }^{1}$.

Vues de l'Europe, vues de l'Amérique du Nord, les sociétés latinoaméricaines sont des sociétés métisses. Certaines d'entre elles le sont de façon marquée, d'autres de façon moins nette, mais l'évocation implicite ou explicite du métissage est toujours présente à leur propos dans l'esprit d'un Européen ou d'un Nord-américain.

À leur propre regard, ces sociétés ont un profil bien différent : la question du métissage n’y entre guère en compte. Ce point de vue concerne la presque totalité

1 Notre propos concerne l'interprétation des apparences physiques comme signe de métissage et non l'utilisation de ce terme dans d'autres domaines (culturel, linguistique...). 
des sociétés de l’Amérique latine, même s’il y a des exceptions. La plus remarquable est certainement le Mexique où, notamment depuis la révolution de 1910, l'identité nationale est fondée sur l'évocation du métissage des origines, sur une méxicanité définie comme le produit de l'union de peuples différents, mais qui garde en mémoire ce qu'étaient ces peuples avant leur rencontre dans le Nouveau Monde.

En effet, de façon très générale, ces sociétés ne se perçoivent pas comme métisses. Les mots «métis », " métissage », " métisser », sont absents du langage quotidien. Ils ne sont présents que dans les propos d'intellectuels, d'anthropologues, de sociologues, alors que dans d'autres milieux on ne ressent pas le besoin de les prononcer. La réflexion que faisait il y a quelques années une étudiante péruvienne à leur propos est particulièrement éloquente : «Au Pérou, ce sont des mots pour un cours d'histoire, qu'on n'entend pas ailleurs ». Ce rappel des cours d’histoire - et plus précisément, des cours d’histoire coloniale de l'école primaire - est récurrent lorsqu'on demande à des Latino-américains dans quel contexte ils les utilisent ou les ont utilisés.

Si ces mots ne se prononcent pas, c'est parce qu'ils ne viennent pas spontanément à l'esprit d'un Latino-américain. De la part de sociétés dont la naissance est marquée par un métissage biologique d’une ampleur inouïe, inconnue ailleurs, on peut se demander à quoi tient cette absence. Cette situation est souvent pensée, de l'extérieur, comme l'occultation, consciente ou inconsciente, de leur « vraie » identité. Or, ne peut-on pas se demander si le fait biologico-historique du métissage est réellement leur fondement identitaire ? Ne serait-ce pas, au contraire, ceux qui le mettent en avant qui se leurrent? 


\section{Le métissage : le contraste entre la définition biologique et la définition sociale}

$\underline{\text { Retour à la table des matières }}$

Une remarque semble ici nécessaire : le métissage étudié par les biologistes n’est pas celui des représentations, qu'elles émanent du peuple ou de chercheurs en sciences sociales. Il importe d'être extrêmement clair à ce sujet car les dérives sémantiques du terme "métissage » conduisent à des confusions qui ne peuvent qu’obscurcir l'analyse.

Partons des fondements historiques du concept de métissage. Ils sont indissociables du concept de « race », la « race » étant entendue au sens d'une entité ayant sa propre essence donc une nature immuable, et le métis étant, dans ce contexte, à la fois le signe de l'altération d'une pureté initiale et un produit inclassable. En démembrant ce concept de la race, la génétique a rénové la vision du métissage. À quoi s'intéresse en effet un biologiste qui étudie le métissage ? À des flux de gènes qui s’échangent entre deux populations en contact, puis au résultat populationnel de cet échange, quelle que soit la distance génétique initiale. Il n'y a pas de différence structurelle, dans cette approche, entre un échange de gènes entre une population d'origine africaine et une d'origine européenne, et un échange entre populations dont les différences ne portent que sur des caractères peu contrastés, par exemple entre des Normands et des Alsaciens.

Or, la perception sociale qualifiera de métissage le premier cas (entre Européens et Africains) mais non le second (entre Européens). En effet, si, du point de vue de la biologie, tout croisement entre populations implique un métissage, il n’en va pas de même pour l'intuition populaire. Chez elle, « métissage » implique qu’il y a croisement entre des « races » définies comme des entités clairement distinctes. En cela, elle garde vivante la pensée classificatrice que l'anthropologie biologique a maintenant abandonnée sous l’influence de la génétique. Il ne faut jamais sous-estimer cette dimension dans l'emploi du terme «métissage » et les implications qu’il véhicule. 
Fait encore plus important : chaque société définit un seuil, valable seulement pour elle-même, qui sépare le métis du non-métis (Benoist, 1992) : tel individu « classé » comme métis dans une société ne le sera pas dans une autre. Par exemple, un Maghrébin issu d'une mère très claire, d'apparence européenne, et d'un père dont les traits du visage et la couleur de la peau témoignent d'une origine africaine, ne sera pas pour autant considéré comme un métis, contrairement à ce qui arriverait dans un cas analogue, à un individu né en France de mère métropolitaine et de père antillais. La comparaison entre les Antilles et la Réunion nous permet de préciser cette question : dans deux sociétés où la conscience du métissage est bien présente, la définition de celui-ci n’est pas univoque. À la Martinique, une personne qui a des ancêtres noirs ne peut pas franchir la ligne de couleur, même si son physique ne permet guère de deviner une origine métisse. Elle sera classée séparément des Blancs à partir de son nom de famille, qui devient un marqueur stable lorsque les apparences voilent les origines biologiques. À La Réunion, l'évidence d'un phénotype ou d'une généalogie se rattachant à l'Afrique n'est pas le critère principal pour l'assignation à une catégorie définie. Cela ne choque personne à La Réunion d'entendre la phrase : «Elle est blanche, mais sa mère est noire ", propos qui traduisent une gestion de l'ethnicité où la souplesse prime sur la rigueur classificatoire. Le message social du " métissage » n’est pas le même dans un cas et dans l'autre: la force inéluctable du premier contraste avec la fluidité du second.

Le métissage n'est donc pas un concept univoque ; chaque société le définit, le façonne, lui donne un contenu, comme elle le fait à propos d'autres faits qu'elle transforme par là en faits de culture, que ce soient le rêve, la naissance d'un enfant, la maladie, le changement des saisons. En parlant de métissage, on est, toujours, dans le domaine des représentations.

Celles-ci s’appuient sur l'apparence physique des individus, que les sociétés interprètent différemment les unes des autres et dont elles infèrent ou non les origines de ces individus. Il est fréquent qu'elles fixent ici ou là des discontinuités entre des groupes selon leur apparence, mais il arrive aussi que l'apparence ne soit pas le critère le plus significatif pour introduire des clivages entre leurs membres.

Or, le fait que le phénotype des individus tienne, au moins en grande partie, à des caractères génétiques donne l'impression que les apparences visibles, relevant d'un fait de nature, ne prêtent pas à des divergences d'interprétation. On a donc 
du mal à concevoir que le poids des représentations soit considérable dans la perception de l'aspect physique des êtres humains. Aussi a-t-on tendance à voir dans le métissage un concept qui relève exclusivement de la biologie et un fait dont l'observation ne saurait être soumise à des variations culturelles, alors qu'en réalité on prend pour observation ce qui est toujours une interprétation.

La façon dont le métissage est envisagé par le biologiste et par l'imaginaire social est donc très contrastée. Or, un point important doit être souligné car il est à l'origine de bien des malentendus. Le discours des intellectuels, que naïvement l'on croit à l'abri des interprétations populaires, a des points en commun avec celles-ci. En effet, contrairement à la définition qu'en donnerait un biologiste, un chercheur en sciences sociales, suivant en ceci le sens commun, parle de métissage là où il "voit » une population qu'il estime issue d'un croisement entre groupes très distincts, et cela, indépendamment de la façon dont les membres de la société étudiée se perçoivent eux-mêmes.

\section{Le métissage en Amérique espagnole}

\section{$\underline{\text { Retour à la table des matières }}$}

La société argentine, considérée aujourd'hui, ainsi que sa voisine uruguayenne, comme la plus «blanche » de l'Amérique latine, est née, comme ce fut le cas de toutes les colonies espagnoles de ce continent, de la rencontre et du brassage de groupes humains d'origine différente et d'apparence contrastée. Les conquérants et colons espagnols, partis pour la plupart seuls, sans famille, pour l'aventure du Nouveau Monde, se sont unis en terre américaine à des femmes indiennes, les femmes espagnoles ayant été très peu nombreuses à participer à l'entreprise de colonisation. Une génération de métis est ainsi apparue dès les

premiers temps de la conquête d'un bout à l'autre des Indes espagnoles. Mais ces métis biologiques ne l'étaient pas socialement. Ayant été assimilés, selon les cas, au groupe de leur père ou à celui de leur mère, ils ont été considérés et recensés, pour leur immense majorité, soit comme des Espagnols, soit comme des Indiens.

Par la suite, le tableau s'est complexifié. D’une part, les esclaves africains, très présents dans certaines régions de l’Amérique espagnole et beaucoup moins 
dans d'autres, sont entrés dans la dynamique de ce métissage. D'autre part sont apparues des catégories et des sous-catégories de métissage, qui se sont affirmées peu à peu. Or, celles-ci ne s'appliquaient pas à l'intégralité des individus de cette nouvelle population née en terre américaine, mais à ceux qui pour des raisons sociales échappaient à la classification entre Espagnols, Noirs et Indiens. Comme ailleurs, cette typologie à base ethnique était solidaire, dans un contexte de conquête, de colonisation et d'esclavage, de la hiérarchie issue de l'ordre politique que la société s'était donné. Mais à la différence d'autres sociétés qui ont connu une histoire analogue, en Amérique espagnole ces catégories étaient suffisamment malléables pour que l'appartenance sociale l'emporte sur la stricte réalité biologique. Ainsi, de façon générale, le terme «métis » s’appliquait seulement à une partie des individus biologiquement métissés : à ceux qui faisaient partie des couches sociales inférieures, mais pas à ceux qui appartenaient aux élites, lesquels, indépendamment de leur apparence physique, étaient reconnus comme des «Espagnols d'Amérique ». La souplesse de la typologie pouvait également permettre à des Indiens, sous certaines conditions, d'échapper à leur catégorie d'origine pour intégrer celle de "métis », ce qui les faisait monter dans l'échelle sociale les libérant, entre autres, des travaux forcés et de l'obligation de payer le tribut. Ces exemples suffisent à montrer que, dès le départ, en Amérique espagnole, la notion de " métis » a été imprégnée par le social, ce qui en a fait, tant qu'elle a été utilisée, une catégorie souple et poreuse.

Or, ces classements n’ont pas survécu au-delà des indépendances : ils ont été oubliés avec la formation des jeunes nations. Alors, ceux qui étaient socialement définis comme Métis et comme Mulâtres sont devenus des nationaux au même titre que ceux que la période coloniale avait connus comme Espagnols d'Amérique, mais ils sont restés dans les couches sociales les moins favorisées, où ils se situaient déjà. Cette évolution a été commune à toute l'Amérique espagnole. 


\section{La société argentine : le souci de l’égalité et la question des apparences}

\section{$\underline{\text { Retour à la table des matières }}$}

Venons-en à l'Argentine et à l'histoire de sa population.

À partir de la seconde moitié du XIX ${ }^{\mathrm{e}}$ siècle, le pays a commencé à accueillir de très importantes vagues d'immigrants. L'Europe apparaissant comme l'image de la civilisation par excellence, à laquelle on souhaitait assimiler les gens du pays, c'est dans ce dessein qu'avait été particulièrement encouragée l'immigration européenne, en accord avec une politique de peuplement du territoire qui avait par ailleurs le souci explicite d'élever le niveau de civilisation de la population locale.

L’immigration en Argentine eut une ampleur inouïe : la population passa de 1200000 habitants en 1857 à 12000000 en 1930. En raison de l’origine des immigrants, la composante européenne augmenta dans de fortes proportions. Cela rendit certainement moins apparents les métissages anciens, qui ne disparurent pas pour autant.

L’esprit de l'époque était parcouru par des courants idéologiquement opposés quant à la question de la prééminence de certains peuples sur d'autres. Il y avait d'une part, certes, l'influence d'une pensée clairement raciale, qui établissait une hiérarchie en plaçant au sommet les capacités intellectuelles des populations dites de race blanche ainsi que leurs productions culturelles. La confusion entre « race » et « culture », fondée sur l'amalgame hâtif entre l'apparence d'une population et les œuvres qu'elle élabore (ce qui par ailleurs est loin d’être une spécificité de l'époque), est fréquente dans les écrits, même lorsque l’idée de supériorité n’est pas explicitement affirmée. D’autre part, l’affirmation de l'égalité entre les hommes prend une ampleur considérable tandis que se généralise l’idée que leurs dissemblances sont le fait de l'éducation et non de la biologie. Selon la théorie évolutionniste, qui fournissait alors un cadre pour penser l'histoire de l'humanité, la distance entre des sociétés marquées par des degrés différents de progrès ne pouvait pas être attribuée à des causes biologiques, les hommes étant considérés 
comme égaux dans leur aptitude à atteindre les stades les plus avancés de civilisation.

C’est ce second courant, celui qui mettait en avant l'égalité des capacités humaines, qui l'emporta dans la pensée des élites politiques argentines de la fin du $\mathrm{XIX}^{\mathrm{e}}$ siècle et qui anima donc les projets qu'elles mirent en œuvre concernant la rencontre de la population locale avec les vagues d'étrangers attirés par l'espoir d'une nouvelle patrie. La notion de hiérarchie raciale n'en était pas pourtant totalement absente : une élaboration singulière lui permit de s'accommoder de cette idéologie qui était avant tout égalitaire. L’univers du «nous » national fut défini et délimité par l'adhésion à la culture européenne, c’est-à-dire à celle de la « race » considérée comme supérieure; mais cela fut pensé de telle façon que l'adoption de cette culture, et l'abandon conséquent des croyances et pratiques d'autres origines (amérindiennes et africaines), impliquait l'inclusion spontanée des individus dans le groupe dit blanc (Quijada, 2000 [2] : 46-47). Aucun obstacle (en particulier celui d'un métissage, qui se dressait en Amérique du Nord ou dans la Caraïbe anglophone et francophone) n’empêchait alors le passage.

Les élites qui ont gouverné le pays durant les dernières décennies du XIXe siècle étaient guidées par la volonté de faire de l'Argentine une nation de citoyens intégrés dans l'égalité. Un premier pas dans ce sens fut, alors que les immigrants affluaient par milliers, la loi de 1884 sur l'enseignement gratuit et obligatoire : elle impliqua la mise à disposition de tous des enseignements de base et l'uniformisation par la langue, ainsi que le modelage identitaire par la transmission des lieux de la mémoire collective qu'une génération d'hommes de lettres et d'historiens s'attachait alors à définir. Ensuite, un jalon fondamental fut l'adoption en 1912 du suffrage universel, qui fit en 1916 d'Hipólito Yrigoyen le premier président en lequel les masses d'immigrants reconnurent leur leader. Mais le point le plus lourd de sens pour la question que nous traitons ici est le processus par lequel la population argentine est devenue, dans son propre imaginaire, homogène quant à sa "couleur ", celle de la population que l'époque considérait comme la plus élevée hiérarchiquement car sa culture incarnait les valeurs alors fondamentales de progrès et de modernité. Ainsi, les Argentins se sont-ils définis comme « une nation blanche et de culture européenne [ce qui est devenu] l'un des principaux axes de l'armature identitaire argentine » (Quijada, 2000 [1] : 10). 
C’est ainsi que s’est développée une « idéologie de la blanchitude », si puissante et si générale qu'elle entraîna véritablement l’oubli de toute l’histoire du métissage dans le pays. Les Argentins finirent par se percevoir comme formant une nation homogène et blanche. Cette idéologie, toujours très vivante, a transformé les regards ; elle a évacué de l'esprit des nationaux la question du métissage, rendant celui-ci invisible aux yeux d'un Argentin alors qu'il n'échappe pas au regard d'un Européen. Dans l'imaginaire national, les particularités physiques sont devenues des traits individuels, et non des éléments à partir desquels on pourrait créer des catégories ${ }^{2}$.

Cette nation « blanche et homogène » a intégré très rapidement des individus d’origine africaine ainsi que, parmi les groupes amérindiens, ceux qui, de gré ou de force, avaient abandonné leur ancienne identité et étaient entrés de façon active dans la vie de la nation. Car l'affirmation que l'Argentine est une nation blanche n’a jamais impliqué l'exclusion de ceux qui ne étaient pas blancs, mais l’ouverture à tous ceux qui étaient prêts à s’intégrer dans le projet historique de la nation. Au sein de cette population «blanche », les différences dans le teint de la peau ne traduisent pas des clivages ethniques. Certes, statistiquement elles sont le signe d'une stratification sociale, les individus d'origine africaine et indienne s’étant majoritairement incorporés à la société en intégrant les couches sociales les moins aisées. Mais individuellement elles ne le sont pas, du moment où les teints les plus variés issus d'anciens métissages se retrouvent aussi bien dans les milieux défavorisés que parmi des membres de la plus haute bourgeoisie de Buenos Aires et, surtout, des provinces.

Cette affirmation de la blanchitude, qui ne repose donc que sur une construction idéologique, est certes contredite par l'histoire ethnique nationale, par l'évidence des brassages entre les populations qui se sont rencontrées sur le terri-

2 La catégorie de « cabecita negra » (lit. : « petite tête noire », nom vernaculaire d'un oiseau migrateur), courante à Buenos Aires, pourrait apparaître comme un désaveu à cette affirmation. Par cette expression, on désigne des individus originaires des provinces, ayant émigré vers la capitale, où ils sont des prolétaires, et dont le teint de la peau témoigne d'un ancien métissage. Cependant, cette dénomination est sociale et non raciale, dans la mesure où elle ne s'applique pas : 1) à ces mêmes individus tant qu'ils sont dans leur province d'origine ; 2) à des individus ayant le même phénotype mais appartenant à une couche sociale plus élevée. Elle a aussi une connotation politique, car dans l’imaginaire national elle a été longtemps liée fondamentalement aux supports du péronisme. 
toire argentin. Elle a néanmoins des conséquences tangibles sur la vie sociale dans la mesure où elle est vécue comme vraie : cette idéologie a construit le regard et lui a appris à voir de l'homogénéité là où d'autres sociétés distinguent des composantes. C'est dans ce sens que le métissage est devenu invisible : n'étant pas porteur de message, n’apportant aucune information significative sur l'individu ou sur la société, il a cessé d’entrer dans le champ de la perception.

\section{Connotations du métissage}

\section{$\underline{\text { Retour à la table des matières }}$}

Pour des observateurs européens ou nord-américains, le métissage argentin et, plus largement, latino-américain, demeure cependant un fait d'évidence, immédiatement visible. Aussi ont-ils du mal à concevoir que des sociétés ayant été largement touchées par des brassages de populations, considèrent le métissage comme non significatif et qu'il demeure pour elles invisible : ils ont souvent vu là une volonté de cacher l'histoire des origines, de refuser une vérité qui dérangerait ces sociétés.

Cette interprétation, qui, sans être nouvelle, s’accorde particulièrement bien avec « l’air du temps », doit être questionnée.

Lorsqu'on évoque un refus, une occultation dans le fait qu'une société biologiquement métisse ne se perçoive pas comme telle, lorsqu'on lui impute une volonté de cacher ses racines biologiques, est-on vraiment un analyste lucide ou bien le porteur d’une idéologie fondamentalement biologisante?

Allons plus loin : celui qui s’acharne à voir un refus ne fait en réalité que mettre l'accent sur les composantes d'origine de cette société métisse au lieu de faire porter son attention sur la nouvelle configuration à laquelle les diverses composantes ont donné naissance. Sa position ne relève-t-elle pas d'un souci excessif de ne pas perdre de vue les « races pures » qui se cacheraient derrière les apparences ? (Benoist, 1996 ; Amselle, 2002).

Il est vrai pourtant qu’on peut penser que si une société biologiquement métisse « oublie » cette condition qui est la sienne, cela peut tenir à un trouble devant le fait de se définir comme un produit hybride, d'accepter pour elle-même l'image 
de ce désordre incertain véhiculée par les représentations du métissage. On se trouve là face à un phénomène bien étudié par Mary Douglas (1966) : le malaise devant le désordre connoté de souillure, devant le désordre vécu comme impur. En effet, il n'est pas aisé pour un groupe humain de se présenter avec un visage évoquant cet entre-deux qui signifie tout d'abord "ni l'un ni l'autre », ce statut ambigu que l'on retrouve au fond de toutes les représentations du métissage, populaires comme savantes, et dont Carmen Bernand (2001) a fait une très fine analyse pour l'Amérique latine.

Mais, au-delà de cet « oubli », il y a peut-être d'autres raisons qui conduisent une société biologiquement métisse à donner d'elle-même une définition qui laisse de côté la question de ses origines.

Ne peut-on pas concevoir des sociétés biologiquement métisses pour qui cette question n'aurait pas de sens dans la mesure où elles font porter l'accent sur ce qu'elles sont devenues plutôt que sur ce qu'elles ne sont plus ? Des sociétés plus attirées par leurs créations que par des soucis étymologiques? Ces sociétés n'auraient pas besoin de mettre l'accent sur le métissage et sur les composantes qui se trouvent à sa base. Bien au contraire, cet accent contredirait le fondement même de leur identité.

Traiter de la société argentine, comme de bien d'autres sociétés latinoaméricaines, sur la base de la notion de métissage semble donc hasardeux. On est face à des configurations sociales où ce qui compte n'est pas ce qui existait avant leur origine, mais ce qui s'est passé depuis leur naissance. De ce point de vue, l'affirmation que j'ai entendue maintes fois dans les profondeurs du monde rural dans la bouche d'individus qui ignorent tout sur leurs origines indiennes et espagnoles : « Nous, on est la race d'ici », est particulièrement significative. C'est leur façon de dire qu'ils sont ce qu'ils sont devenus. 


\section{Connotations de la créolité}

$\underline{\text { Retour à la table des matières }}$

Tournons-nous alors vers la réalité sociale argentine non pas à partir de l'angle du refus ou de l'oubli, mais en tenant compte de la façon dont la société se définit elle-même, une société qui, en même temps qu'elle se sent blanche et de culture européenne, se définit comme «criolla».

Le terme «criollo », de même que sa traduction française, «créole », désignait au départ les enfants d’Européens nés sur le nouveau continent. La population colonisatrice venue de la métropole espagnole ayant été très largement masculine, les Créoles ont été, dès le départ et dans une grande majorité, des enfants d'hommes espagnols et de femmes indiennes : des métis. Cependant, dès lors qu’ils étaient rattachés au groupe social de leur père, ils étaient définis comme Créoles et faisaient donc partie des Espagnols : «Criollo» signifie, en effet, « Espagnol d'Amérique ». On sait la place que prit, dans toute l’Amérique espagnole, l’identité créole au moment des indépendances, quand il devint indispensable, pour les Créoles, de se démarquer des Métropolitains. Avec la naissance des nations, certaines sociétés latino-américaines eurent tendance à utiliser de moins en moins le terme « criollo » pour nommer leur population ou une partie de celleci, et elles ne le gardèrent que pour se référer, dans chaque pays, à des choses « de chez nous », telles qu'un type de musique ou de cuisine. Mais dans d'autres sociétés, telle l’Argentine, « criollo » a continué à s’appliquer à la population.

La permanence de cette dénomination dans ce pays a été liée au besoin ressenti par les nationaux de se démarquer, dans une première phase, de l'importante vague d'immigrants arrivés à partir de la deuxième moitié du XIX ${ }^{\mathrm{e}}$ siècle. Rappelons que la politique d’immigration de l'époque avait, parmi ses objectifs, celui d’européaniser la société argentine, ce qui revenait, selon les critères évolutionnistes de l'époque, à la "faire monter » dans l'échelle de la civilisation. Or, si la culture européenne était vue, d'Argentine, comme un sommet à atteindre, très vite le décalage devint évident entre l'image de raffinement qu'on avait de l'Europe et l'image qu'en donnaient ceux de ses représentants qui débarquaient alors au port de Buenos Aires. Face au nombre impressionnant de ces nouveaux arrivants, que 
la misère et la faim avaient chassés de chez eux, face à ces immigrants dépourvus de tout prestige social, les gens du pays se sont identifiés comme «criollos » pour se distinguer d'eux. Ils ne tenaient pas à être confondus avec l'ensemble des étrangers de l'Ancien Monde qui, par-delà leurs appellations nationales respectives, reçurent le terme générique de « gringos ». Entre criollos et gringos se dressa une frontière nourrie d'un mépris réciproque : les uns ne manquaient pas de remarquer la rusticité des gringos, les autres jugeaient qu'un criollo ne valait pas un Européen.

Mais cette coupure ne dura pas longtemps. Par la suite, les efforts politiques d'uniformisation en vue de faire percevoir l'Argentine comme une nation homogène et blanche prirent une autre direction, ce qui aboutit non pas à européaniser la population locale, comme le voulait le projet initial, mais à « créoliser » la population immigrée. L’Argentine devint ainsi une nation de Créoles, ce qui est largement sous-estimé par la plupart des chercheurs.

Nous touchons là à un point important : il n’y a pas de contradiction, pour un Argentin, entre « être une nation blanche » et « être une nation de Créoles ». Même si, au départ, la plupart des Créoles étaient biologiquement métis, le métissage s'est complètement effacé derrière une créolité qui est devenue la façon d'être blanc en Argentine.

La notion de créolité traduit ainsi la volonté d'être, avant tout, des nationaux, en laissant de côté la question des origines ; celle-ci ramènerait à une réalité antérieure à la création de la société argentine telle qu'elle se reconnaît aujourd'hui et elle empêcherait donc de mettre en évidence l'unité de la société actuelle. En ce sens, parler de métissage plutôt que de créolité va à contre-courant d'une réalité essentielle et significative : en Argentine, percevoir des métis là où il y a des créoles ne peut être le fait que d'un regard autre qu'argentin. Mais si la mise en avant de l'identité criolla caractérise l'Argentine, des processus homologues ont eu lieu dans la plupart des pays d'Amérique latine où, même lorsque la population ne se définit pas par le terme "criollo », la conscience du métissage a été également évacuée. 


\section{Quelques hypothèses}

$\underline{\text { Retour à la table des matières }}$

En conclusion, nous sommes conduits à formuler quelques hypothèses sur cette idéologie.

Apprendre à ne pas voir le métissage relève certes d'une position idéologique en accord avec le projet de nation mis en œuvre en Argentine à la fin du XIXe siècle. Mais on est en droit de s’interroger sur la nature de l'idéologie qui veut à tout prix débusquer le métissage et conduire non seulement à le percevoir mais à en faire un élément fondateur de l’identité.

Certaines analyses des sociétés latino-américaines, qui mettent l'accent sur le métissage, semblent extrapoler à leur propos ce que révèle à cet égard la dynamique sociale antillaise, dont la réalité est bien différente malgré les apparences. Mais, au-delà de ces généralisations abusives, ne peut-on pas se demander si l'insistance sur le métissage, qui place au premier plan les racines, les composantes préalables au mélange, ne traduirait pas la vision de sociétés où la distinction entre pureté et impureté raciale serait significative ? S’agirait-il, alors, d’une façon subtile de distinguer des sociétés « pures » du point de vue racial et des sociétés qui ne le seraient pas?

Dans l'analyse d'une identité sociale, ce n’est pas la vérité biologique des origines qui compte, mais la vérité sociale qui soutient la construction identitaire. Or, nous constatons l'importance grandissante que prend depuis peu une idéologie biologisante dans divers domaines de la vie sociale, parmi lesquels la question de l'ethnicité. Même lorsqu'il est entendu dans un sens non biologique, le « métissage » porte en lui la notion souvent trouble du mélange d'essences différentes, dont la convergence fait une société intrinsèquement différente d'une société non métissée. Cette idéologie irait-elle jusqu’à envahir même le choix des questions que se posent les chercheurs en sciences sociales, le point de vue qu'ils privilégient au moment d'envisager un fait de société ? Ne choisissent-ils pas, en mettant le métissage en avant, de donner prééminence aux « racines » des sociétés sur les forces qui les intègrent? 
On peut aussi se demander quelle sera, sur les sociétés latino-américaines elles-mêmes, l’influence de la progression d'une idéologie selon laquelle la définition des groupes sociaux semble indissociable de leur origine et qui devient l'outil d'une amplification planétaire des revendications identitaires. Des leaders politiques sud-américains sont entrés récemment dans un discours racialisant, qui trouve un écho croissant chez des anthropologues du continent. Je m’écarte ici un instant de ma fonction qui est celle d'analyser un fait social pour exprimer l'espoir que la créolité argentine ne disparaîtra pas derrière un métissage qui un jour deviendrait visible.

Dernière remarque, à laquelle j'attache une grande importance. Penser et exprimer le métissage latino-américain dans un discours qui renvoie aux racines, suscite chez un latino-américain un certain scepticisme, voire certains soupçons. On lui présente une idéologie de fragmentation, d'essentialisme des origines, à laquelle il trouve bien des point communs avec ce que la pensée coloniale exprimait dans les discours sur les tribus. Ne touchons-nous pas là au cœur négligé du débat sur le métissage?

\section{RÉFÉRENCES}

$\underline{\text { Retour à la table des matières }}$

Amselle, Jean-Loup (entretien avec), «Le métissage : une notion piège », dans Nicolas Journet (coord), La culture. De l'universel au particulier, pp. 329333, Ed Sciences Humaines, 2002.

Benoist, Jean, « Le métissage : biologie d'un fait social, sociologie d'un fait biologique », dans Métissages, linguistique et anthropologie, tome II, pp 13-22, L’Harmattan, 1992.

Benoist, Jean, « Métissage, syncrétisme, créolisation : métaphores et dérives », Etudes Créoles XIX (1) : 47-60, 1996.

Bernand, Carmen, «Mestizos, mulatos y ladinos en Hispanoamérica : un enfoque antropológico de un proceso histórico », dans M.L Portilla (coord.), Motivos de la antropología americanista. Indagaciones en la diferencia, pp. 105133, 2001. 
Quijada, Mónica, « Introducción », in Mónica Quijada, Carmen Bernand et Arnd Schneider, Homogeneidad y nación. Con un estudio de caso : Argentina, siglos XIX y XX, pp. 7-13, Madrid, CSIC, « Tierra nueva e cielo nuevo », 2000 (1).

Quijada, Mónica, «El paradigma de la homogeneidad », in Mónica Quijada, Carmen Bernand et Arnd Schneider, Homogeneidad y nación. Con un estudio de caso : Argentina, siglos XIX y XX, pp. 15-55, Madrid, CSIC, « Tierra nueva e cielo nuevo », 2000 (2).

Douglas, Mary, De la souillure, Paris, La Découverte, 2001 [1966].

Sturzenegger-Benoist, Odina, L’Argentine, Karthala, « Méridiens », 2006.

\section{Fin du texte}

